\title{
Surgical Excision of Unusual Cervical Cyst
}

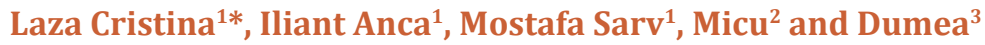 \\ ${ }^{1}$ ENT surgeon, University Ovidius, Europe \\ ${ }^{2}$ Department of Anatomo-pathology, Europe \\ ${ }^{3}$ Infectious Disease Clinic, Europe
}

Submission: August 27, 2017; Published: September 07, 2017

*Corresponding author: Cristina Otilia Laza, ent/omf clinic-scju constanta, University Ovidius, Constanta -B-Dul Tomis 145, Europe, Email: cristinaotilia@gmail.com

\begin{abstract}
Hydatidosis is a parasitic infection caused by the larvae of the tapeworm Taenia echinococcus. Men are an accidental host of the parasite. Most common form of presentation is a cystic lesion -embrio transformation developed in the lungs, liver, heart, brain, bones. We present a rare case with a neck cyst, finally identified like hydatid, primary and single sign of the disease. Our patient was known with the laterocervical right cyst diagnosed as 4 year before like branchial type. When the patient decide to accept the surgery, we didn,t consider necessary new studies -lab test or imagistics because nothing change just usual test required by surgery and general anaestasia. The diagnosis was an intraoperator surprise, and confirms anatomo-patology. The treatment consist in pericystectomy-complete excision of the cyst, isolate the surgical field with betadine and saline, anti anaphylaxis shoch measures followed by antihelmintic treatment with Albendasole.After the surgery we start to study serologic ,imagistic the patient but we didn't discover another site. No recurrence was discovered till now. The purpose of this paperwork is to demonstate that even in non endemic areas hydatic cyst can be considered in the differential diagnosis a cystic neck mass.
\end{abstract}

Keywords: Cystic neck swelling; Taenia echinoccocus; Neck dissection

\section{Introduction}

Cystic Echinococcosis:Echinococcosis is a disease caused by the development inside the viscera, especially in the liver and lungs, of larvae of Taenia echinoccocus. Man is an accidental host of the parasite, the animals playing the role of host [1]. Three types of Taenia Echinococcus have been identified that may cause the disease to occur:

\section{Echinococcus granulosus,}

\section{Echinococcus multilocularis and}

\section{Echinococcus oligartus}

The life cycle of the parasite (Echinococcus granulosus) requires definitive hosts and intermediate hosts. Final hosts are normally carnivores, such as dogs, while intermediate hosts are usually herbivores, such as sheep and cattle. People are accidental hosts for the parasite because they interrupt the cycle of parasitic infections. The disease cycle begins when adult tenia infects the intestinal tract of the definitive host (most commonly the dog). Adult Tenia produces eggs (ca. 600-800) which are eliminated in the faeces. Intermediate hosts, usually herbivores, become infected after ingestion of the parasite eggs (sheep and cattle can be infected when they are grazing on contaminated faeces from dogs containing these parasite eggs).
These eggs are very resistant and can survive in the external environment for about a year. After the eggs have been ingested from the contaminated grass, they reach the inside of the sheep's intestines and then (the larval stage) penetrates the intestinal wall and is transported by blood to various parts of the body, such as the liver and lungs, where slow cysts develop hydatid. These cysts can grow quite large and contain many young tears floating in a clear liquid (Figure 1).

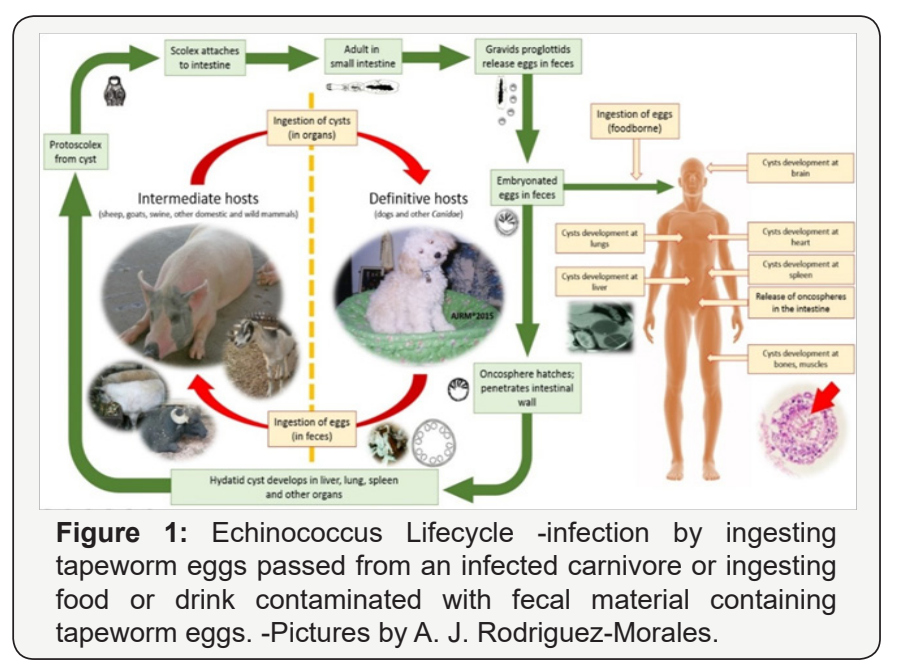


If a dog is then fed with meat from the infected animals, cysts are ingested and the cyst wall is digested in the gut, releasing protoscolecsi that turn into about two months in adult skin in the dog's intestine. Within the dog's intestine tenia produces eggs, which are then eliminated in feces. People can become infected with eggs eliminated in the dog's feces (for example, the dog licks the face after cleaning himself). Eggs can also contaminate food and water or other objects, such as collar. Ingestion of tanned eggs can occur through the consumption of contaminated food. Risk factors for the development of hydatid disease are primarily contact with animals that are the intermediate hosts: pigs, sheep, cattle, dogs, wolves, coyotes. . The professions exposed are butchers, pastors, farmers, animal breeders.-larval cystic stage of Echinococcus granulosus [2].

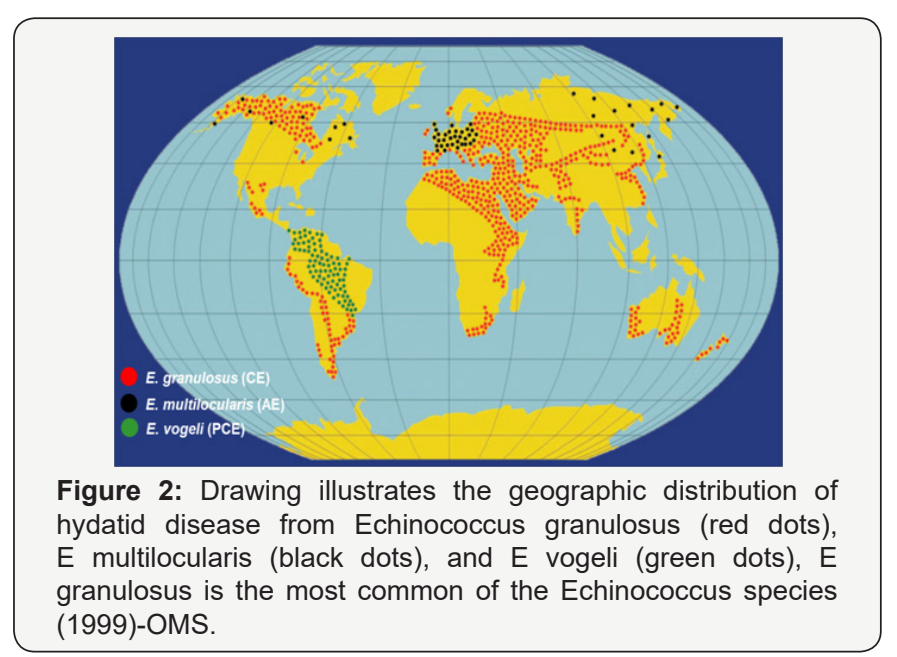

The eggs hatch after digestion of the outer capsule in the intestinal mucosa and the larvae penetrate the mucosa, reaching the liver through the portal vein. Most of these embryos become lodged in the hepatic sinusoids, where they either die or develop into one or several hydatid cysts. A systemic spread will occur if the larva passes through the capillary sieve. Hydatid cyst, develops most frequently in the liver (65\%), the lungs (25\%), out of remaining $10 \%$ occurs in muscle, spleen, bones, kidneys, brain, eye, heart, and pancreas .A hydatid cyst is extremely rare in head and neck region even in geographical areas where ecchinococcal infestation is frequent. Only a few cases of hydatid cyst located in neck have been reported (Figure 2).

\section{Structure of the Hidatid Cyst}

Echinococcus granulosus tapeworm cyst is $1-1.5 \mathrm{~cm} /$ year in the larval stage can develop in any organ: $-80 \%$ solitary cyst $2 / 3$ liver, the second most common organ is the pulmonary. The cyst is made by two layers

a. Laminated -external layer -pericyst-periparasitic host tissue

b. Germinal -internal layer-multipotential germinal layer giving rise to the brood capsules and protoscolesces, inside the cyst is filled with clear fluid, brood capsules and protoscoleces the growth rate is $1-1.5 \mathrm{~cm} /$ year
Symptoms are caused by the interaction between the adjacent organ and the cyst

\section{A. Complications}

i. cyst rupture and spread of protoscolexes and larval material through vessels

ii. bacterial cyst infection,

iii. immunological response such as asthma, anaphylaxis, nephropathy (Figure 3).

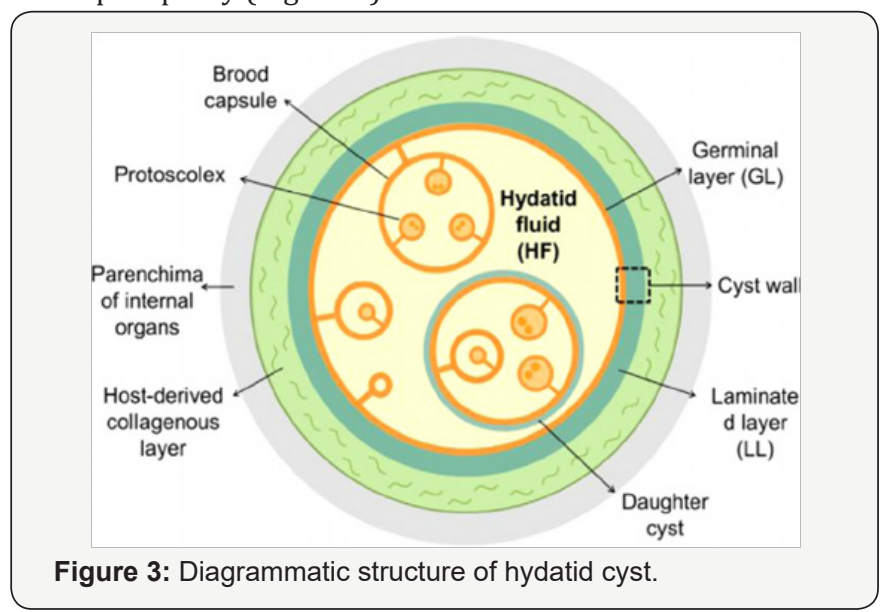

\section{B. Evolution}

Some cysts can grow or persist without a change for many years; others may spontaneously or traumatically break or collapse, disappearing completely with spontaneous healing $[3,4]$.

\section{Case Report}

The patient M.N. 37 years of age from the rural environment was admitted in our clinic with a left, 4-year, clean, stable, laterocervical oval mass, in the anterior triangle of the neck exactly bellow the angle of the mandible. A neck swelling can have several different diagnosis depending in the age of the patient, location of the neck, duration, symptoms, clinical evolution, A swelling on the neck in an young adult is a common presentation as a branchial cyst, external laryngocelae, lymphangioma flebectasia of the IJV a metastatic cystic lymph nodes regional metastasis of an cancer primary located on the palatine tonsils or rhino pharynx [5].

Clinical Examination: History-patient has no complains like fever, pain difficulties to swallow or to breath.

A. Inspection /palpation -revealed a $8 / 10 \mathrm{~cm}$ in size, ovalar laterocervical right mass in bellow the angle of the mandible, at the anterior border of the SCM. The mass covered with normal skin .was soft, fluctuant, nontender, and nonreductible. There were no other mases on the neck or in other parts of the body and the larynx, trachea were not displaced [6].

B. Ent examination -complete-was normal (Figure 4). 


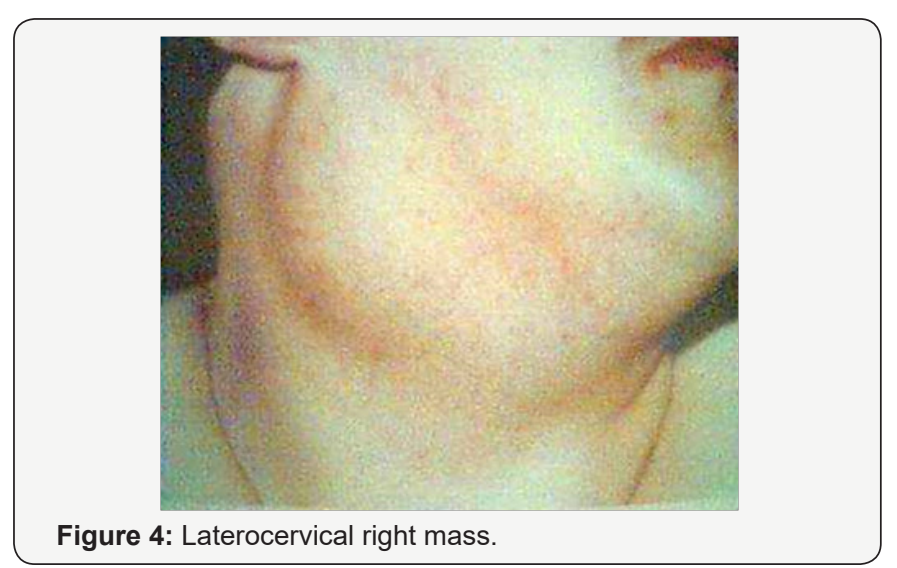

C. Treatment: Because we already have CT -scan of the neck studies, and there were not much differences in the aspect of the mass-just a little bit larger, we sustain the diagnosis of branchial cyst and we discuss with the patient about the surgery. So we prepare the patient for surgery with the usual lab tests and X-ray lung, EKG-cardiovascular exam, anesthesiology exam [7].

In surgery we use a cervicotomy with incision along the anterior border of the SCM muscle .We discover a large cystic regular ,oval mass but very adherent to surround tissues ,but we think this can be caused by the repeated infections of the neck cyst in the time without a treatment. Because was very large usually in branchial cyst surgery we use to reduce the sizes of the cyst making a puncture and removing a part of the content than we use a forceps to seal the puncture point. It was a total surprise when we saw leaking clear fluid and multiple whitish vesicles suggestive of hydatid daughter cysts, so immediately we seal the puncture, also prevent anaphylactic shock. Consequently, the entire operating field was washed and protected with hypertonic saline solution, betadine. After this incident we remove carefully and complete the cyst in fact was a complete pericistectomy. Drainage and wound closure layer by layer were like usual [8].

We send the mass to the hystopathological exam and suspicion of hydatid cyst was confirmed intraoperative by the pathology department. The final pathology examination reported laminated cyst wall and vesicles, indicative of hydatid cyst. We start postoperatory an intens study to exclude a lung ,liver brain cyst or another more important location of an hydatidosis [9-11].

\section{Specific Laboratory Analyzes}
I. TGO, TGP,CCT ,LDH
II. Total, Direct, Indirect Bilirubin,
III. FA
IV. CBC- HLG-Eosinophilia -15\%,
V. hipogammaglobinemia

VI. specific antibodies by screening tests: indirect hemagglutination test and the enzyme-linked immunosorbent assay (ELISA) have a sensitivity of $80 \%$ overall (90\% in hepatic echinococcosis, $40 \%$ in pulmonary echinococcosis)

VII. confirmatory test Immunodiffusion and immunoelectrophoresis demonstrate antibodies to antigen 5

VIII. Puncture with a needle of the cyst with microscopic examination- large risk of anaphylactic shock or dissemination

IX. biopsy needle under anthelmintic useful coating in the differentiation of malignacy and abscesses

The Casoni Test is a skin test in which the body's reaction to the intradermal injected hydatid antigen is established. Currently, it is largely abandoned due to its reduced sensitivity (70\%), low accuracy and increased risk of severe local allergic reactions. After surgical extraction the antibodies persist in the blood for one year [11,12].
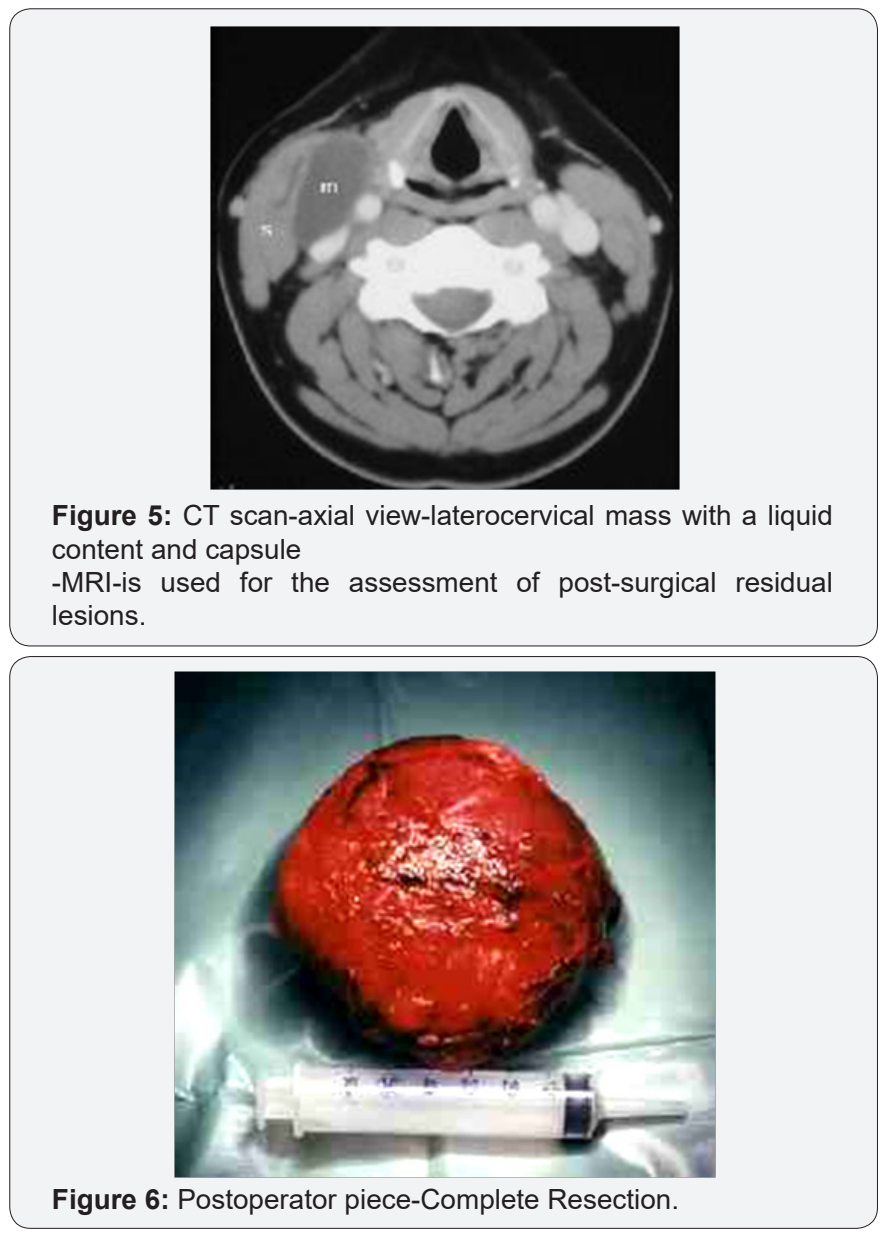

Imaging Tests: Radiography (the pulmonary is nonspecific and largely irrelevant, the hydatid cyst appears as a round opacity with uniform density.) The presence of a thin layer of calcification that delineates the cyst is suggestive of the hydatid 
cyst. Abdominal ultrasound - liver cervix Computed tomography has an accuracy of 98\%. CT cysts are well defined, have thin or thick walls, and old cysts have hydatid sand, highlighted as a layer of different density. It is the best test for the differentiation of the hydatid cyst from the amoeba or bacterial abscess, carcinoma and hemangioma CT must explore brain, thorax, abdomen, pelvis (Figure 5). Because we can't find nothing we consider the neck site unique, primary even extremely rare localization of a hydatic cyst, also because of the contamination of the wound with content of the cyst we use ALBENDASOLE for a month.The cervical wound heal perfect with a very fine scar and there was no recurrence of the hydatidosis till now ,on periodic follow -up (Figure 6)

\section{Discussion}

a. Hydatid disease is a parasitic infection caused by the larva Equinococcus granulosus, endemic in Mediterranean countries, South America, the Middle East and Asia. Hydatid cyst at unusual sites have been reported around the world including the spleen, kidney, heart, bones, muscles and cranium, Hydatid disease in cervical region is rare, with only a few cases reported in the literature .At this site, an isolated infestation with no history of cystic rupture elsewhere in the circulation, as seen in our case, is particularly unusual.

b. Hydatid cysts are usually not considered in the differential diagnosis of head and neck cystic swellings, especially in non-endemic areas in the absence of hydatid disease elsewhere in the body. The rarity of the disease in this anatomical location presents a diagnostic difficulty for the physician if he or she is not familiar with the disease.

c. During its life cycle, the dog is its definitive host; the adult larva sets its eggs in the dog's intestines and the environment is then contaminated upon expulsion outside with the host's faeces. Intermediate hosts can be sheep, cats and, occasionally and accidentally, people, who become infected by ingesting contaminated food; the eggs then hatch in the duodenum. At that point, the parasite spreads by portal circulation and reaches the liver (the most frequently involved organ, $75 \%$ of the time, followed by the lungs, $15 \%$ ); however, other routes have been described, such as porto-cava anastomosis, lymphatic circulation or direct egg inhalation, which could explain less frequent locations such as the spleen, kidneys, heart, central nervous system and even soft tissue, as in the case that we present here.

d. Head and neck hydatid disease is rare, with less than $0.5 \%$ being reported, including cases in the parotid gland, thyroid and parapharyngeal area. The neck location is limited to countries where echinococcosis is endemic and in patients with antecedents of hepatic and/or lung echinococcosis. It is very rare to find it as the primary presentation of hydatid disease.

e. Hydatid cysts in the neck are usually asymptomatic and slow growing, with occasional pressure symptoms.
Spontaneous or iatrogenic cyst rupture can have serious consequences, including anaphylactic shock, even as the first symptom. Personal and family history, as well as the individual's profession and place of residence, can help to suspect the diagnosis; however, the characteristic signs and symptoms are not pathognomonic in the head and the neck, making its diagnosis a real challenge.

f. Radiological studies are of great relevance, particularly echography, CT2 and NMR, with the NMR scan being the study of choice if there are complications or presence of a solid pattern. These tests are generally more sensitive than serological diagnosis. Consequently, the presence of internal septa or membranes floating in a cyst-even in the presence of negative serological results is considered to be the pathognomonic criterion for hydatid cyst.

g. The disease is specifically diagnosed by analysing the fluid content of the cyst; however, despite this, the use of fine needle biopsy is controversial, given the potential risk of spreading the eggs and of causing an anaphylactic reaction from cyst rupture.

h. Serological studies have low sensitivity and specificity, so a negative result does not exclude the disease. However, recent studies have shown that ELISA results are very important in follow-up of patients in treatment. No serological test was done in our case because hydatid cyst was not in the clinical differentials. MRI done was not able to do exact characterization and the report was suggestive of benign cyst.

i. The differential diagnosis therefore includes branchial cleft cyst, bronchogenic, thymic, thyroid, parathyroid, thoracic duct Zenker-esophageal diverticulum, lymphangioma, flebectazia of IJV, laryngocaele, benign tumor that is congenital and acquired cystic lesions of the neck-like methastasis.

j. For the evaluation of mass lesions in the cervical region, fine-needle aspiration cytology (FNAC) is beneficial however due to possibility of an anaphylactic reaction, dissemination of disease and recurrence as result of spillage of cyst contents, it is not recommended in the routine evaluation of suspected hydatid cysts. FNAC was not performed in our case due to possibility of benign cystic disease; the case was subjected to surgical excision.

k. When hydatid disease is found, a complete systemic examination should be carried out to rule out involvement of other organs. The diagnosis of Echinococcus infection mainly depends on the clinical history of the patient, diagnostic radiological findings and serologic tests. ELISA, Casoni skin tests, latex agglutination, immunoelectrophoresis and direct hemagglutination are serological methods, used for the diagnosis of hydatid disease. Sensitivity of serology is high (80-100\%) for liver cysts, but low for lung (50-56\%) and other organs (25-56\%). Importance of these tests lies 
mainly in the follow up of treated patients. An increase in titer indicates recurrence of disease and a decrease in titer indicates resolution .Imaging modalities like US, CT and MRI remain more sensitive than serodiagnosis. These techniques help to determine the cystic avascular nature of the lesion. Daughter cysts, vesicles and internal septa can also be demonstrated.

l. The treatment of choice is still surgery, making sure the germ layer of the cyst (producing the protoscolices) is removed. If the cyst ruptures, the surgical field should be surrounded by dressings soaked in hypertonic saline solution to avoid dissemination.

m. A pericystectomy (removal of the cyst with its outermost fibrous layer) is normally performed. Therapy with imidazole derivatives, fundamentally albendazole, is recommended as complementary treatment before and after surgery. Medical treatment may also be preferred if surgery is not possible, as in patients with multiple organ involvement, patients with a cyst in an inaccessible location, or patients with poor general condition and having unacceptable risk for surgery, Standard therapy consists of 3 cycles of 4 weeks each, with serological and hepatic monitoring due to the hepatotoxicity of this drug. A new therapeutic strategy, called PAIR, has been used for cases of hepatic or pulmonary involvement. This option consists of the percutaneous aspiration, injection and aspiration of the cyst content, associated or not with intra-cystic application of imidazoles. Although the technique, still being developed, seems to offer fewer complications and relapses, for head and neck involvement is advised against, given the lack of experience with it in these cases. Combination of medical therapy with imidazole derivatives has been used in the management of patients with recurrence and high-risk contamination [1215].

\section{Conclusion}

Hydatid cyst with a head and neck site is very rare in countries where echinococcus infestation is not endemic, but still the physicians mast be well informed and capable to take such a cyst in consideration when is confronted with a neck cyst mass imaging techniques, though sensitive sometimes cannot pin-point the exact etiology of cystic lesion. During surgical removal of cysts in which no definite etiology has been made preoperatively, great care must be taken to avoid spilling of the cystic contents [16].

\section{References}

1. WA Webb, L Mc Daniel, L Jone (1984) Foreign bodies of the upper gastrointestinal tract: Current management. South Med J 77(9): 1083-1086.

2. JK Hamilton, DE Potter, Philadelphia (1993) Gastrointestinal foreign bodies. In: Gastrointestinal disease: Pathophysiology, Diagnosis and Management, In Sleisenger MH, Fordtran JS, WB Saunders (eds): 286292.

3. GJ Herrantz, VJ Martinez, SA Garcia (1991) Esophageal foreign bodies in adults. Otolaryngol Head Neck Surg 105: 649-654.

4. Vele DD, Dubey SP (1997) An unusual foreign body: a whole fish in the throat. Auris Nasus Larynx 24(2): 207-209.

5. Deidiker R (2002) Return of the killer fish: accidental choking death on a bluegill (Lepomis macrochirus) American Journal of Forensic Medicine and Pathol 23(2): 197-198.

6. Kalan, M Tariq (2000) Foreign bodies in the nasal cavities: a comprehensive review of the aetiology, diagn. pointers, and therapeutic measures. Postgrad Med J 76: 484-487.

7. MM Sangeeta, RS Greval, D Singh (1999) Paediatric nasopharyngeal foreign bodies. Indian J Otolaryngol Head Neck Surg 51(1): 80-82.

8. PK Majumdar, AK Sinha, PB Mookherje, SN Ganguly (1999) An unusual foreign body (10 N.P. Coin) in nasopharynx. Indian J Otolaryngol Head Neck Surg 52(1): 93.

9. SC Sharma, S Bano (1992) An unusual foreign body in nasopharynx. Indian J Otolaryngol Head Neck Surg 1: 42-43.

10. JH Faraj (1993) Upper airway obstruction by ascaris worm. Can J Anesth 40(5): 471-482.

11. B Singh, EL Gady Har, M Kantu (1997) Complications associated with 327 foreign bodies of the pharynx, larynx and esophagus. Ann Otol Rhinol Laryngol 106(4): 301-304.

12. FO gut, M Bereketoglu, C Bilgeric, S Totan (2000) Metal ring that had been lodged in a child's nasopharynx for 4 years. Ear Nose Throat J 80(8): 235-236.

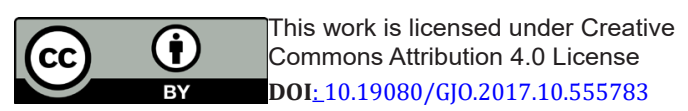

\section{Your next submission with Juniper Publishers will reach you the below assets}

- Quality Editorial service

- Swift Peer Review

- Reprints availability

- E-prints Service

- Manuscript Podcast for convenient understanding

- Global attainment for your research

- Manuscript accessibility in different formats

( Pdf, E-pub, Full Text, Audio)

- Unceasing customer service

Track the below URL for one-step submission https://juniperpublishers.com/online-submission.php 